\title{
Características democráticas en videojuegos de simulación de gobierno
}

Democratic Characteristics in Government Simulation Video Games

Características Democráticas em Videogames de Simulação de Governo
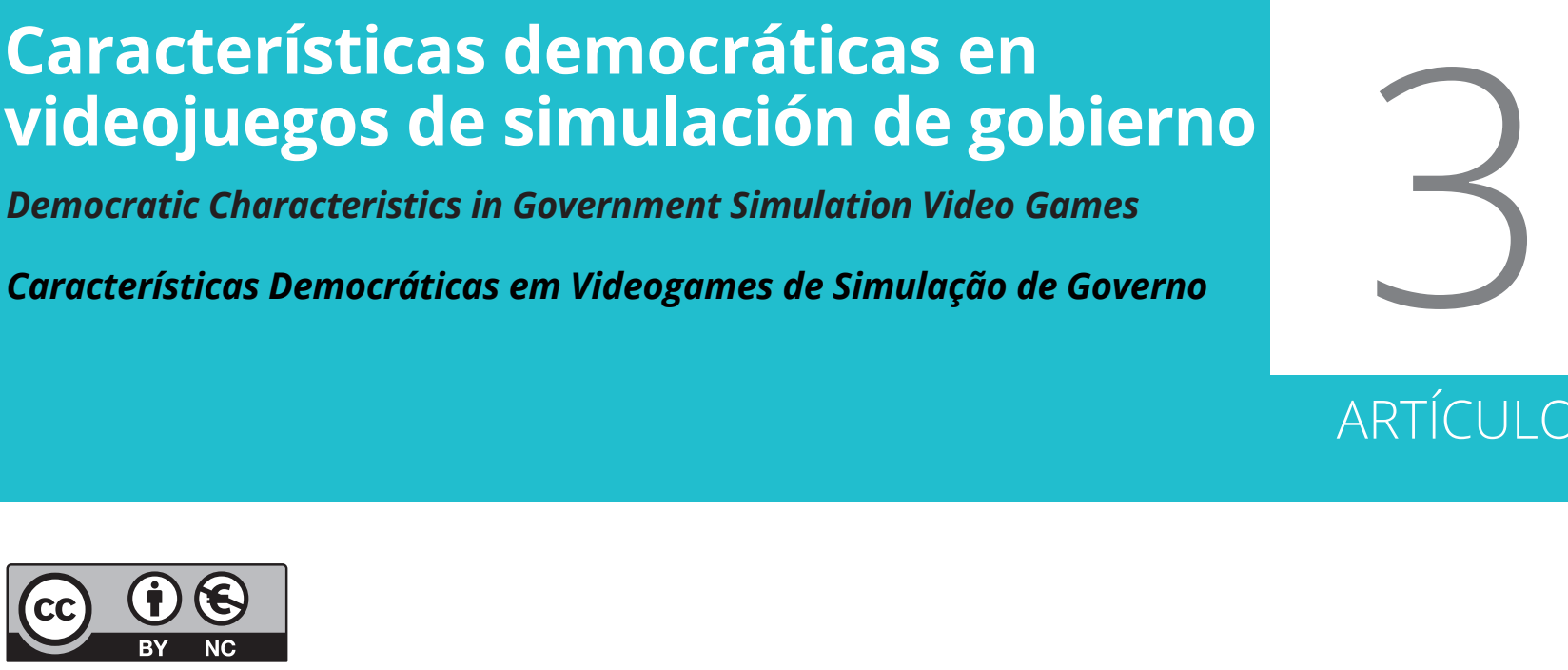

\section{Carlos Moreno Azqueta}

Universidad de Castilla-La Mancha (España)

Investigador predoctoral en formación, especialización en game studies.

carlos.moreno.azqueta@gmail.com

orcid.org/0000-0001-5771-3069

RECIBIDO: 01 de octubre de 2021 / ACEPTADO: 11 de enero de 2022

\section{Resumen}

Este artículo analiza nueve videojuegos de gestión de una comunidad política, estudiando las características democráticas presentes en la relación entre el jugador y los ciudadanos virtuales. Las comunidades políticas de los juegos se presentan en ocasiones como homogéneas y estructuradas en torno al líder, y en otras, como plurales y contestatarias. Los juegos muestran diversos mecanismos de contrapoder ciudadano informal, pero el conflicto puede presentarse como político y legítimo, o enmarcarse en un problema de productividad o criminalidad. Finalmente, se observan importantes déficits en las dimensiones de deliberación y participación en la toma de decisiones, para lo cual se proponen algunas mecánicas alternativas.

\section{PALABRAS CLAVE}

Videojuegos, Democracia, Simuladores de gobierno.

\section{Abstract}

This paper analyses nine videogames centered on managing a political community by studying the democratic characteristics present in the relationship between the player and the virtual citizens. The political communities of the games are sometimes presented as homogeneous and structured around the leader. In other cases, they appear as plural and unsubmissive. The games show diverse mechanisms of informal citizen counterpower, but the conflict may be presented as political and legitimate, or as a productivity or crime problem. Finally, we note 
important deficits on the dimensions of deliberation and decision-making participation. Based on these, some alternative mechanics are proposed.

\section{KEYWORDS}

Videogames, Democracy, Government simulators.

\section{Resumo}

Este artigo analisa nove videogames de gestão de uma comunidade política, estudando as características democráticas presentes na relação entre o jogador e os cidadãos virtuais. As comunidades políticas dos jogos se apresen- tam ora como homogêneas e estruturadas em torno do líder, ora como plurais e rebeldes. Os jogos mostram diversos mecanismos de contrapoder do cidadão informal, mas o conflito pode ser apresentado como político e legítimo, ou enquadrado em um problema de produtividade ou criminalidade. Por fim, observam-se déficits significativos nas dimensões de deliberação e participação na tomada de decisões, para os quais se propõem algumas mecânicas alternativas.

\section{PALAVRAS-CHAVE}

Videogames, Democracia, Simuladores de governo.

\section{INTRODUCCIÓN}

En la introducción a su tesis doctoral dedicada al análisis videolúdico, Pérez Latorre (2010) recurre al planteamiento de Brian McHale (1987) en el que defiende que la ciencia ficción es el género fundamental de la postmodernidad. Lo es, argumenta, porque la ciencia ficción se centra en la exploración de un nuevo mundo, con entidades y reglas propias, más que en el seguimiento de las aventuras de los personajes. Al igual que la ciencia ficción, los videojuegos contienen esa doble dimensión narrativa, en la que la comprensión del mundo videolúdico representa una parte fundamental de la experiencia de juego. Estos mundos, al mismo tiempo, son impresionantes conglomerados de contenido ideológico, que nos hablan de nuestra realidad y la moldean a través de realidades ficcionales.

Los simuladores de gobierno son videojuegos en los cuáles los jugadores toman el control de una comunidad política y gestionan su desarroIlo, tomando habitualmente decisiones políti- cas de relevancia. Al igual que la ciencia ficción, presentan "mundos posibles" (Planells, 2015), y simulan sistemas políticos complejos, que en ocasiones buscan asemejarse a nuestros sistemas contemporáneos —como en Democracy 3 (Positech Games, 2013) - y en otras se sitúan en escenarios de fantasía o ciencia ficción, llegando a manejar comunidades políticas no humanas - como sociedades de pacíficos dragones, entre otras, en Endless Legend (Amplitude Studios, 2014)-. En unos u otros casos, ofrecen representaciones particulares de la política y el papel de los ciudadanos en la comunidad, y pueden ofrecer imaginarios alternativos respecto a cómo deberíamos organizarnos y vivir. Jugar a estos juegos es una práctica democrática, en el sentido de un ensayo, un aprendizaje político particular que debe tener en cuenta tanto el contenido diseñado del juego como la interacción y las emociones e ideas suscitadas en los jugadores.

En este artículo realizaremos un análisis de contenido de nueve videojuegos de simulación de gobierno con el objetivo de explorar las re- 
presentaciones que estos ofrecen sobre sus sistemas políticos virtuales, y estudiar qué relación guardan estas con conceptos que tradicionalmente se han asociado a la democracia.

Nuestra idea de democracia proviene de una larga tradición histórica de conceptos, instituciones y regímenes políticos, un conglomerado de ideas en muchas ocasiones contradictorias entre sí, pero que circulan unidas en nuestro imaginario popular. Se trata de uno de esos "significantes flotantes" (Laclau, 2016), que diversos actores buscan apropiarse. Lo hacen, por otra parte, porque la idea de democracia aporta legitimidad: en España, en 2021, casi el $80 \%$ de los españoles consideraba que la democracia era "preferible a cualquier otra forma de gobierno" (Centro de investigaciones sociológicas, 2021); en ese sentido, afirmar que algo es, o no es, democrático, es un argumento poderoso.

Al mismo tiempo, nuestras sociedades contemporáneas viven una cierta crisis, o degradación, de sus instituciones democrática. La idea de la "desafección política", una pérdida de confianza hacia las instituciones y en particular hacia los partidos, lleva presente décadas (Mair, 2015), pero puede verse acrecentada con nuevas dinámicas preocupantes, como el surgimiento de movimientos y partidos autoritarios o la creciente difusión de noticias falsas (estas últimas contribuyen a construir visiones del mundo radicalmente distintas, en las que no hay verdades comunes sobre las que debatir, lo cual nos lleva a conflictos irresolubles).

Esta situación invita a una reflexión colectiva sobre nuestras instituciones, de la cual los videojuegos pueden formar parte y este artículo busca ser una modesta contribución. Como veremos, muchos de los videojuegos analizados no buscan representar sistemas políticos democráticos, pero pueden incorporar elementos presentes y característicos de una democracia. Partimos entonces de una noción de democracia no como régimen político puro, sino como conglomerado de características que pueden estar presentes en diversos regímenes. En las páginas siguientes plantearemos algunas características de lo que a lo largo de la historia se ha considerado democracia y que buscaremos en los sistemas políticos presentes en los videojuegos analizados. Antes, sin embargo, presentaremos las inspiraciones teóricas de este trabajo desde la perspectiva de los game studies.

\section{MARCO TEÓRICO}

\subsection{GAME STUDIES Y SIMULADORES DE GOBIERNO}

Las investigaciones sobre el contenido político de los videojuegos adquieren sentido al asumir que estos tienen un efecto sobre las ideas y cosmovisiones de sus jugadores. La sociología ha trabajado desde hace tiempo sobre la idea de que los productos culturales contribuyen a formar nuestra representación del mundo, y los game studies han señalado algunas particularidades de los videojuegos (como la interacción o la capacidad de construir mundos con sus propias reglas) que potencialmente refuerzan su impacto social y político (Bogost, 2007). Algunos estudios (Imhoff et al., 2021; Barthel, 2013) han querido evaluar con mediciones concretas los aprendizajes políticos de los jugadores después de jugar a determinados videojuegos, a través de estudios experimentales o de encuestas, con resultados modestos pero positivos. Se registraban determinados aprendizajes con niveles de validez estadística suficientes, pero es probable que muchos de esos aprendizajes no sean claramente conscientes, o se desarrollen más a través del juego conti- 
nuado que en un estudio experimental temporalmente limitado. El análisis sociocultural, del que los videojuegos forman parte, se mueve en un terreno pantanoso, con indicadores claros de su importancia y de los efectos políticos de la cultura, pero una dificultad evidente para medirlos con claridad en una sociedad donde el consumo cultural es extremadamente variado y plural.

Aunque los estudios de contenido de videojuegos se han centrado, en general, en la crítica política (Pobłocki, 2002; Zamaróczy, 2017, entre otros), más recientemente los game studies han empezado a prestar atención a los videojuegos como instrumentos de promoción de valores cívicos y éticos. La literatura sobre ética $y$ videojuegos ha desmentido algunos mitos sobre jugadores sádicos, mostrando que estos ponen en juego habitualmente comportamientos morales (Schrier, 2016), y señalando componentes que el diseño de un juego puede tener en cuenta para generar reflexiones éticas (Schrier, 2015, Zagal, 2011, Simkins, 2008). Otros han señalado como algunos videojuegos pueden ayudar a promocionar valores cívicos (Dishon \& Kafai, 2019) o reflexionar sobre la administración pública (Exmeyer \& Boden, 2020). En una línea más crítica, la revista gamevironments publicó un número en 2020 centrado en las relaciones entre democracia y videojuegos. Esto nos lleva a pensar que la forma en la que los simuladores de gobierno involucran a los jugadores en la gestión de un Estado y en la interacción con los ciudadanos virtuales podría tanto reforzar ideas autoritarias sobre la realidad social como incentivar comportamientos cívicos y prodemocráticos, dentro y fuera del videojuego.
Por otra parte, aunque los simuladores de gobierno raramente se tratan como género, existen múltiples análisis comparativos o de juegos individuales que resaltan y critican sus características políticas (por ejemplo: McNeil, 2016, Pobłocki, 2002; Shields, 2009). Los temas concretos que los autores resaltan son variados: imperialismo y colonialismo (Douglas, 2002, Ford, 2016, Oliva et al., 2009), desarrollismo ecocida (Evans-Thirlwell, 2021) o relaciones internacionales (Zamaróczy, 2017), entre otros. Unos pocos, sin embargo, se centran en características relacionadas directamente con la organización del sistema político y las interacciones entre la ciudadanía y el poder de Estado. Planells (2015), por ejemplo, sugiere que Tropico es una interesante representación del pluralismo político, en tanto que los ciudadanos se organizan en grupos ideológicos (ecologistas, capitalistas...) con intereses y "mundos ideales" distintos; de esa forma se pone de relieve tanto la homogeneidad o pluralismo de la comunidad política representada como la capacidad de los ciudadanos de apostar por determinadas políticas públicas. Respecto al mismo juego, Shields (2009) habla de la organización de un cierto "contrato social" entre jugador y ciudadanos que surge de la capacidad de estos últimos de boicotear su gobierno. Por su parte, Dolkemeyer (2020) presenta un análisis más estético para Frostpunk, en el que sugiere que existen mecanismos, no presentes en otros juegos, por los cuáles el jugador se siente dentro de la comunidad política, con lo que maximiza potencialmente el componente empático. 


\subsection{CARACTERÍSTICAS HISTÓRICAS DE LA DEMOCRACIA}

Nuestra concepción de la democracia es producto de milenios de experimentación política, instituciones y conceptos. Más que hablar de "democracia", tiene sentido hablar de "características de la democracia", aspectos que en un momento $u$ otro han formado parte de su historia política. Estas características serán los elementos que buscaremos en los juegos, con lo que explicaremos brevemente cómo han operado a lo largo de la historia.

\section{- Participación en la toma de decisiones}

Tradicionalmente, se considera la Atenas clásica como la primera democracia (aunque existan experiencias anteriores). Para Josiah Ober (2018, pp. 57-76), el elemento fundamental de la democracia ateniense se encontraba en el acceso de los ciudadanos corrientes a las posiciones de poder y a la toma de decisiones, ya fuera a través de la asamblea (abierta a todo ciudadano) o las instituciones escogidas por sorteo (como los tribunales populares o la bou(é). La toma de decisiones por parte del pueblo era el elemento constitutivo de la democracia griega, pero ha desaparecido mayormente en otros regímenes considerados democráticos, dónde se ha impuesto la selección popular de líderes para que sean estos quienes tomen las decisiones.

\section{- Deliberación pública e intereses colecti- vos}

Otro aspecto significativo de la Atenas clásica era la importancia dada a la deliberación, un mecanismo que ponía en juego y entrenaba la virtud cívica, presente en todos los ciudadanos (Platón, 2013). La deliberación ha encontrado su espacio en todos los regímenes políticos democráticos, pero con enfoques distintos: si bien en Atenas, o en lo que se ha venido a llamar la tradición republicana, la deliberación tenía que ver con la virtud cívica y los intereses colectivos, para los liberales tiene que ver con la negociación entre intereses distintos en búsqueda de una solución que satisfaga a la mayoría. La deliberación ha perdido peso político desde las primeras experiencias democráticas, pero en las últimas décadas la corriente de la democracia deliberativa, defendida particularmente por Habermas (1998), ha defendido recuperarla, normalmente unida a una mayor participación popular en la toma de decisiones. Se han puesto en marcha algunas experiencias de democracia deliberativa a través de la selección de un número reducido de ciudadanos por sorteo para la elaboración de propuestas complejas (como una nueva legislación electoral en Canadá), con resultados alentadores (Lang, 2007)

\section{- Representación y selección de líderes}

Aunque la representación ya estaba presente en Atenas (a través de unos pocos puestos electivos, pero sobre todo del sorteo y la idea de la asamblea como "pueblo reunido"), ha sido la piedra angular de las democracias liberales, que rechazaron el sorteo en favor de la elección por dos motivos fundamentales: seleccionar a líderes considerados "mejores" que sus representados (en lugar de "iguales"), y entender la legitimidad política no como un producto de la participación en la toma de decisiones, sino como un producto del consentimiento (Manin, 1998, pp. 103-118). Así pues, nuestras democracias representativas se basan en la selección de representantes y líderes políticos, en lo que Schumpeter (1971) calificó como un proceso de selección de élites competitivas. 


\section{- Contrapoder y división de poderes}

Por otra parte, tanto en el liberalismo como en la tradición republicana se han privilegiado mecanismos de contrapoder para evitar la tiranía. En su mayor parte estos mecanismos han sido institucionales, como la división de poderes o las limitaciones legales, pero a lo largo de la historia se han desarrollado también, en todo tipo de regímenes, contrapoderes ciudadanos no institucionalizados (protestas, boicots, huelgas...).

Por último, algunas otras características formarán parte de nuestro análisis. Varios autores han defendido un cierto nivel de igualdad económica como un atributo propio de la democracia, en tanto que asegura derechos ciudadanos y una mejor distribución del poder en la sociedad (Tusell Collado, 2015, pp. 183-184). Por otra parte, la teoría política ha discutido mucho respecto a las características que debería tener una comunidad política en términos de homogeneidad social o pluralismo; los liberales siempre han defendido su idea de comunidad como una asociación de individuos, mientras que autores como Schmidtt han apostado por articulaciones menos instrumentales (Schmidtt, 1998).

\section{METODOLOGÍA Y SELECCIÓN DE MUESTRA}

La muestra está compuesta por nueve de simuladores de gobierno: Faraón (1999), Imperivm Civitas III (2009), Civilization V (2010), Tropico 4 (2011), Democracy 3 (2013) Endless Legend (2014), Frostpunk (2018), Surviving Mars (2018) y Surviving The Aftermath (2020)' . Estos son simuladores relativamente populares, con importantes números de ventas en comparación con otros juegos del mismo género y con una distribución temporal que, privilegiando los juegos más modernos, incluye algunas obras antiguas. Al mismo tiempo, la mayor parte de estos son juegos de construcción de ciudades, pero dos de ellos (Civilization y Endless Legend) son considerados 4X (acrónimo de Explore, Expand, Exploit, Exterminate; juegos de construcción de imperios y búsqueda de la supremacía). Finalmente, Democracy simula la toma de decisiones sobre políticas públicas en un Estado nación moderno.

Desde una perspectiva metodológica, múltiples modelos de análisis se han propuesto a la hora de estudiar los videojuegos, considerando sus particularidades respecto a otros objetos culturales (Pérez Latorre, 2010; Navarro, 2016), y nos apoyamos en ellos. Nuestra comprensión del jugador que ocupa la posición del Estado se nutre de una perspectiva semiótica, similar a la propuesta por Pérez Latorre (2010), pero el conjunto de nuestro análisis se enmarca en lo que podríamos definir como análisis "sistémico" o "ludológico". Esto se debe a que queremos centrarnos en el funcionamiento interno del sistema político, entendido como un conjunto diseñado de instituciones, actores y reglas de juego (institucionales y no institucionales). Queremos conocer quién toma las decisiones, cómo y mediante qué procesos, para lo cual ese acercamiento parece el más adecuado. Los aspectos narrativos son fundamentales para comprender cómo se presentan esas reglas e intuir la visión que los jugadores podrían

1 A continuación, estos juegos se citarán sin detallar el año ni la entrega particular (Imperivm Civitas en lugar de Imperivm (ivitas III), para facilitar la lectura y porque las conclusiones son normalmente aplicables a la saga en su conjunto. 
tener de ellas, pero ese análisis se reservará para otra ocasión.

Más concretamente, nuestro análisis del sistema político se centra en cuatro ejes:

- Las competencias de actuación de los ciudadanos y sus reglas de activación (Pérez Latorre, 2010, p. 120), véase, lo que estos pueden hacer, y cómo y por qué lo hacen (en representación del poder de los ciudadanos y sus repertorios de acción colectiva).

- Las competencias de actuación del jugador (que en este caso representa el poder del gobernante-Estado).

- Las características de la comunidad política, como la composición social de los ciudadanos o la distribución de recursos.

- Los objetivos del juego.

El análisis de estos elementos videolúdicos, a su vez, se organizará en tres apartados desde el punto de vista de la teoría política: las características de la comunidad política, los sistemas de contrapoder ciudadano, y las dimensiones de deliberación y participación directa en la toma de decisiones.

\section{ANÁLISIS DE JUEGOS}

\subsection{CARACTERÍSTICAS FUNDAMENTALES DE LA COMUNIDAD POLÍTICA}

Las comunidades políticas virtuales de los juegos analizados presentan un conjunto de características relativas a su configuración política básica, lo que podríamos llamar su carácter. Estas parecen moverse en una línea con dos tipos ideales en los extremos: por una parte, nos encontramos con comunidades orgánicas, con fuerte homogeneidad social, un líder natural y una suerte de destino manifiesto expresado en los objetivos de la partida (como Endless Legend). Por otra, con comunidades heterogéneas, en las que los ciudadanos presentan intereses diversos y el liderazgo se pone en duda más habitualmente. En términos generales, los juegos que presentan comunidades totalmente ficticias se asemejan más al primer modelo, mientras que aquellas que proponen representaciones históricas supuestamente fidedignas (Imperivm Civitas) o relativamente modernas se acercan más al segundo.

A pesar de que la selección popular de líderes ha sido considerada un elemento fundamental de las democracias modernas, en los simuladores de gobierno la partida empieza con el jugador ya en la posición de líder-Estado. Sin embargo, el tipo de liderazgo que se presenta es variable. En Civilization, por ejemplo, el líder se presenta como personificación del carácter de la comunidad, de forma que ambos se confunden; es, además, un líder inmortal, eterno, presente durante los aproximadamente 6000 años de partida, una ficción videolúdica que nos presenta un modelo de comunidad política de tintes schmittianos (Schmitt, 1998). En casos menos radicales, el líder es el fundador de la comunidad (como en Surviving The Aftermath) lo que le otorga un cierto "destino manifiesto" en ocasiones relacionado con la supervivencia en un mundo peligroso. En otras, el líder dirige la comunidad, pero la usa con cierta lógica patrimonial, como en Imperivm Civitas, donde la fundación de ciudades tiene como objetivo el crecimiento de la influencia de la familia. Finalmente, Tropico y Democracy destacan porque sus liderazgos son los más disputados: deben presentarse a elecciones competitivas, y perderán la partida de no ganarlas. 
Esa línea entre la organicidad y la pluralidad parece representarse también en los objetivos. En Endless Legend, por ejemplo, existen misiones "de facción", relacionadas con el carácter cultural de cada una de las razas de fantasía jugables, mientras que en Civilization se asume una cierta voluntad natural en la humanidad de llevar a su pueblo "a la gloria" (que normalmente viene a significar la supremacía). Al otro extremo se encuentran, de nuevo, Tropico y Democracy, donde los objetivos de las jugadoras se encuentran en gran medida indeterminados (aunque hay objetivos formales, se puede optar por políticas públicas muy distintas con impacto en la comunidad) y donde además los ciudadanos tienen objetivos propios y diferentes entre sí. Ambos juegos se basan en sistemas de facciones, donde los ciudadanos pertenecen a determinados grupos ideológicos y desean políticas y formas de sociedad diferentes (Planells, 2015).

Ese elemento convierte a Tropico y Democracy en los juegos con mayor nivel de heterogeneidad social. Cabría tal vez citar también a Imperivm Civitas, donde la comunidad se encuentra necesariamente dividida en clases sociales, que tienen intereses distintos respecto al tipo de bienes y servicios que desean. Estos tres juegos son también los que presentan mayores niveles de diferencias económicas, aunque en los dos primeros una política de redistribución puede reducirlas. En los demás, los intereses y planteamientos ideológicos de los miembros de la comunidad son muy similares o, como mínimo, la sociedad se expresa siempre como un todo unificado y orgánico. Esto último es interesante, por ejemplo, en Frostpunk, donde el juego presenta discusiones entre los ciudadanos respecto a la política del jugador, pero en última instancia la opinión pública se ve reflejada y unificada en los indicadores de descon- tento y esperanza de la comunidad, y cuando el jugador se ve expulsado nunca se presenta un conflicto civil, sino un acto de la comunidad en su conjunto. Incluso si pensamos que es una decisión de la mayoría, la comunidad se presenta como un todo unificado a través de esa decisión.

Por otra parte, existen algunas divisiones sociales funcionalistas (como los especialistas en Civilization, o los biólogos o geólogos en Surviving Mars), pero estas raramente incluyen diferencias ideológicas ni tampoco diferencias en la redistribución de recursos (en Endless Legend o Civilization, por ejemplo, nunca encontramos ningún tipo de acaparamiento de recursos, y en Faraón el acceso a estos no depende de si los ciudadanos pueden pagarlos, si no de si su vivienda se encuentra en una zona de provisión; los ciudadanos, de hecho, nunca emplean su dinero para nada que no sea pagar impuestos, pero se van de la ciudad si estos son demasiado altos).

Así, pues, un primer acercamiento al tipo de comunidades políticas nos ofrece diversos modelos. Nos encontramos con comunidades fuertemente estructuradas, con líderes naturales y un carácter colectivo reflejado en sus objetivos; también con comunidades instrumentales, fundadas por los líderes pero que estos emplean de acuerdo con sus objetivos propios, y en las que habitualmente los ciudadanos llegan o abandonan la ciudad en función de su atractivo y las condiciones de vida que ofrezca, como si se tratara de un intercambio monetario. Y, por último, existen también algunas comunidades plurales, heterogéneas, más similares a nuestra visión moderna de la sociedad. Aunque cada juego suele acercarse más a una u otra, estos son tipos ideales, y es posible que cada juego contenga características de otros. 
El siguiente diagrama busca mostrar visualmente las características de los juegos analizados en base a dos de las dimensiones planteadas, la de homogeneidad social (que se refiere a la similitud de las ciudadanas entre sí en términos de intereses y actuación, y que normalmente viene acompañada de una similar distribución de recursos) y el nivel en el que el liderazgo del jugador se ve disputado. Cabe destacar que, dado que cada juego presenta características únicas y difícilmente equiparables entre sí, las posiciones de los juegos no deben tomarse de forma estricta, si no más bien indicativa de tendencias generales.

Figura 1

Diagrama de distribución de los juegos por homogeneidad social y liderazgo disputado

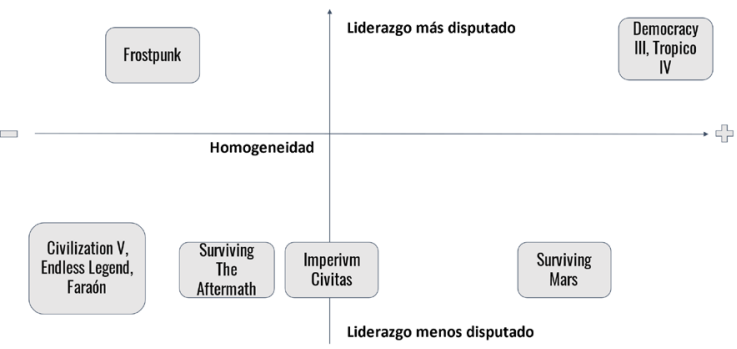

\subsection{CONTROL Y CONTRAPO- DER}

Todos los sistemas políticos históricos que se han considerado democracias han incorporado una dimensión legal e institucional, un conjunto de reglas de juego que definían lo que podía hacerse y cómo. Los procedimientos legales, de hecho, han sido considerados muchas veces contrapoderes frente a las pretensiones de actores poderosos, con capacidad fáctica para hacer cosas que las leyes no les permiten.

Los simuladores de gobierno, sin embargo, apenas incorporan mecanismos legales que regulen el funcionamiento de la vida política.
Es cierto que los juegos presentan "leyes" propias que regulan su mundo, pero estas, incluso cuando son sociales, se parecen más a las físicas: definen lo que se puede o no se puede hacer de modo absoluto, y no únicamente formal. Los actores institucionales externos al jugador son extremadamente raros: no existen jueces ni parlamentos, sino solo figuras como los "consejeros" en Civilization (sin ningún poder real) o los ministros en Democracy, que nunca toman decisiones por su cuenta, sino que ponen su capital político al servicio de los jugadores. Solo en casos como Tropico pueden cometerse ilegalidades (como asesinar a un rival político), pero incluso allí no se encontrará con la oposición de actores institucionales, sino de los propios ciudadanos.

En un contexto de ausencia general de instituciones, reglas de juego y procesos de selección de líderes, la oposición ciudadana se convierte en el elemento fundamental de contrapoder en los juegos analizados, y este es a su vez el componente democrático más desarrollado de entre los estudiados.

Al igual que para los liberales, que entendían el control popular más como un sistema de incentivos que de obligaciones (Manin, 1998, pp. 201-206), en los simuladores de gobierno abundan los mecanismos por los cuales el descontento popular tiene implicaciones negativas para el jugador, pero raramente le obliga a tomar determinadas decisiones. Los juegos varían en cuanto al poder de control de los ciudadanos: en algunos casos pueden expulsar al jugador del poder y hacerle perder el juego (Democracy, Frostpunk...), mientras que en otros el efecto del descontento es menor, como el hecho de que algunos ciudadanos abandonen la ciudad (Faraón). La intensidad del control suele venir de la mano de otra diferencia rele- 
vante, relativa a la concepción del conflicto que se presenta. En ese sentido, se presentan cinco formas de concebir los efectos del descontento:

\section{- El descontento como problema de pro-} ductividad. En estos casos, el descontento ciudadano no genera ninguna respuesta política propiamente dicha, pero tiene efectos perniciosos sobre la comunidad política y representa un obstáculo a la victoria del jugador por generar un problema de ineficiencia. En el constructor de ciudades Faraón parte de la población abandonará la ciudad si pierden el acceso a bienes o servicios, lo cual dificulta el crecimiento y el desarrollo de las economías de escala. En Civilization la infelicidad de la población disminuye su crecimiento y provoca que los soldados luchen peor, y en Endless Legend se ve afectada la productividad.

\section{- El descontento como problema de delin-}

cuencia. En algunos juegos, como Surviving The Aftermath o Imperivm Civitas, el descontento provoca que algunos ciudadanos se vuelvan delincuentes y roben comida o incendien edificios, respectivamente.

\section{- El descontento como problema de ges-} tión empresarial. Este enmarcado no tiene la presencia y consistencia de los otros cuatro, pero encuentra cierta expresión en Surviving Mars, un juego de construcción de una colonia en Marte. Antes que bienestar, a los habitantes de la colonia se les proporciona "confort" a través de la provisión de servicios. Al mismo tiempo, los turistas visitan habitualmente la colonia y puntúan su satisfacción con la experiencia al volver a la Tierra. Se adopta de esta forma una gestión empresarial del descontento, donde los incentivos políticos adquieren el vocabulario y la lógica de la gestión privada de los servicios, y los ciudadanos se convierten en clientes.

- El descontento como ira política. En este caso, el descontento genera un malestar propiamente político contra el gobierno y sus políticas, y se expresa en la voluntad de expulsarlo del poder, pero no se construye sobre propuestas políticas alternativas concretas; es el caso de Frostpunk.

\section{- El descontento como alternativa políti-}

ca. Tanto en Tropico como en Democracy 3, los ciudadanos que expresan su descontento tienen preferencias políticas claras alternativas a las del jugador (como, por ejemplo, una menor o mayor defensa del medio ambiente o la inversión en políticas sociales). Al contrario que en los casos anteriores, el descontento no surge solo de malas condiciones de vida, sino también de un desacuerdo político.

Estos son tipos ideales, por lo que cada juego puede combinar diversas perspectivas, aunque no suelen hacerlo. En los dos últimos tipos el descontento se plantea como un problema político, y no meramente técnico, pero es el quinto el que claramente aporta mayor agencia a la ciudadanía, cuyas preferencias se acercan al mismo nivel cognoscitivo que las del jugador. Los tres primeros, por el contrario, se acercan más a la idea de la política como tecnocracia, donde la eficiencia en la persecución de los objetivos del juego sustituye a la política propiamente dicha. 
El siguiente diagrama resume la posición aproximada de los juegos basándose en tres dimensiones: el poder de los ciudadanos para marcar la agenda política del jugador (en la línea horizontal), la institucionalización del conflicto político, a través por ejemplo de elecciones regladas (en la línea vertical) y el enmarcado del conflicto, que puede observarse en los colores de cada juego: productividad, en verde; delincuencia, en negro; gestión empresarial, en azul; descontento como ira política, en rojo y descontento como alternativa política, en morado. Como comentábamos, podemos observar correlaciones entre el enmarcado del conflicto y el poder de los ciudadanos, así como entre la institucionalización y el poder ciudadano.

\section{Figura 2}

Diagrama de distribución de los juegos por capacidad de actuación de los ciudadanos, institucionalización de conflicto político y enmarcado del conflicto político

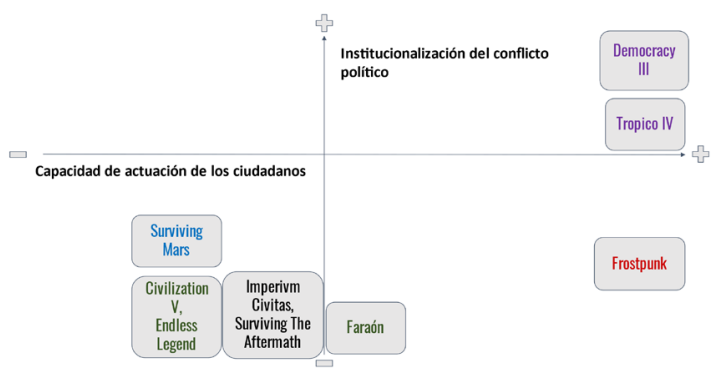

\subsection{DELIBERACIÓN Y PARTICIPACIÓN EN LA TOMA DE DECISIONES}

La deliberación ha sido un aspecto fundamental para el proceso de toma de decisiones entre diversas tradiciones democráticas, ya sea entendida como un proceso de entrenamiento y puesta en práctica de la virtud cívica para alcanzar una sabiduría colectiva, o como un proceso de negociación entre facciones que permite alcanzar la solución satisfactoria para la mayoría (este último es el caso para los liberales (Manin, 1998). Si bien la deliberación hoy parece más bien formal a nivel parlamentario, esta ocupa un papel relevante en la toma de decisiones y, sobre todo, en la formación de la llamada "opinión pública". En nuestras democracias complejas, la deliberación y la formación de la opinión pública se da a través de la mediación de una multitud de actores con distintas funciones y poderes, desde individuos y personajes públicos a movimientos sociales, organizaciones y partidos, y medios de comunicación. Como resultado, encontramos una sociedad con una opinión pública dinámica, en la que diversos temas entran y salen de la agenda pública y las percepciones sobre estos de los ciudadanos y otros actores cambian con el tiempo. También ofrece el que es, a priori, el principal aliciente para la actividad política, que es la capacidad de presentar ideas y proyectos de sociedad y convencer a los actores necesarios para ponerlos en marcha. La deliberación funciona también como mecanismo de contrapoder, motivo por el cual los regímenes autoritarios suelen querer limitarla.

La deliberación es, sin embargo, una ausencia fundamental de los simuladores de gobierno. En los juegos, los ciudadanos reaccionan a las situaciones en las que se encuentran ellos o la comunidad (por ejemplo, su felicidad en Civilization o la economía en Democracy), o a las decisiones tomadas por el jugador (de forma unánime o heterogénea), pero esas reacciones se encuentran prefijadas y programadas, nunca se debaten ni cambian por ser convencidos de algo. 
En ocasiones la deliberación se presenta brevemente de forma narrativa, como en Frostpunk, cuando después de aprobar una ley aparecen opiniones variadas de los ciudadanos respecto a ella, pero sin ningún efecto mecánico, o como el discurso en Tropico, en el que a través de promesas el jugador logra votos para las elecciones, pero no convence a nadie de cambiar su postura ideológica. Al mismo tiempo, en Democracy los ciudadanos pueden cambiar de opinión con respecto a un tema concreto si cambia su situación social (por ejemplo, si un conductor deja de conducir, pasará a no tener problemas con las subidas de impuestos al combustible), pero nunca como efecto de un proceso deliberativo, sino como consecuencia de un cambio de intereses objetivo. De esa forma, la ausencia de deliberación supone una ausencia de dinamismo social y una aproximación a la democracia y a los ciudadanos como electores/consumidores racionales, cuyas decisiones se toman de acuerdo con la evaluación de sus intereses y preferencias. La democracia se representa de forma similar a como la imaginaban los teóricos de la democracia económica, basada en la teoría de la elección racional (Downs, 1957).

Uno de los potenciales efectos de esta representación de la democracia tiene que ver con la promoción de reacciones autoritarias en el juego. Ante la imposibilidad de convencer a los ciudadanos de una política determinada, la única forma de llevarla a cabo es ejercer unilateralmente el poder para ponerla en marcha $y$, en algunos casos, como en Frostpunk, despojar a los ciudadanos de sus herramientas de contrapoder para evitar que lo impidan. Esta dinámica puede reforzarse en el caso de que el jugador tenga la seguridad de que lo que pretende hacer es objetivamente mejor que lo que proponen los ciudadanos, lo que nos lleva a plantearnos la cuestión de la idea de sabiduría colectiva y la infantilización de la ciudadanía en los simuladores de gobierno.

Como hemos comprobado anteriormente, solo en dos de los juegos los ciudadanos proponen políticas alternativas a las de los jugadores, mientras que en los demás estos reaccionan a las circunstancias, pero carecen de programa. Al mismo tiempo, los juegos pueden presentar circunstancias críticas para la supervivencia (como en los escenarios postapocalípticos de Frostpunk y Surviving The Aftermath) u objetivos del juego que el jugador conoce, pero no queda claro si los ciudadanos comparten (como la supremacía en (ivilization). En ambos casos, los ciudadanos pueden aparecer como poco colaborativos, poco comprensivos hacia el jugador, e incluso caprichosos en sus demandas. Del mismo modo, el jugador dispone de un conjunto de informaciones técnicas a las que los ciudadanos no pueden acceder: desde datos estadísticos de productividad y condiciones de vida, desarrollos futuros de tecnología y sus requisitos actuales, o potenciales desastres naturales o cambios meteorológicos graves. Esta información le permite elaborar una estrategia a largo plazo (frente a las necesidades a corto plazo de los ciudadanos), lo que, unido a la falta de iniciativa política de estos últimos, contribuye a ese proceso de infantilización en el que los jugadores aparecen como los actores ideales para la toma de "buenas" decisiones.

Algo similar ocurre con la idea de sabiduría colectiva. Los defensores de la deliberación han planteado que es un proceso adecuado para la toma de buenas decisiones, ya que, entre otras cosas, involucra más perspectivas sobre la cuestión que debatir que la del autócrata. 
El problema, de nuevo, es que no existe una asamblea donde los ciudadanos pongan en común esas perspectivas, sino que el único actor con posibilidad de conocerlas (en la medida en que el juego se lo permita) e incorporarlas en la decisión final es el propio jugador (por ejemplo, leyendo las reivindicaciones de cada facción en Tropico).

Así, pues, la ausencia de mecanismos deliberativos, acompañada de una representación de los ciudadanos como consumidores racionales y sujetos infantiles al mismo tiempo, y la concentración de la información en manos del jugador promueven la aceptación de una relación autoritaria como la fórmula más eficaz y adecuada para la toma de decisiones en el juego. Los propios juegos dan entonces una justificación ideológica al hecho de que no exista un solo mecanismo de participación directa de los ciudadanos en la toma de decisiones. Los edificios que se construyen, las leyes que se firman, las relaciones internacionales, la tecnología que se investiga y cualquier otra decisión pública, que pone en juego los recursos comunes de la comunidad, es tomada exclusivamente por el jugador-Estado.

Aunque existen claras dificultades mecánicas y técnicas para la simulación de la deliberación y la participación en el juego, estas podrían dar lugar no solo a juegos muy innovadores, sino también a juegos con capacidad de enseñar valores prodemocráticos. En la expansión de Frostpunk "el último otoño" aparece una mecánica teóricamente muy interesante: al enfrentarse a las reclamaciones laborales de los obreros, el jugador puede crear un sindicato, que reduce el riesgo de huelga, pero en el cual los trabajadores se reúnen y toman algunas decisiones para la comunidad de forma autónoma e inde- pendiente 2 . Otros juegos podrían incorporar mecánicas significativas en las cuales los jugadores cedieran parte de su poder de decisión y obtuvieran, a cambio, mayor colaboración ciudadanía, promoviendo la negociación y cesión por encima de la aplicación de la autoridad.

\section{CONCLUSIONES}

En términos generales, los simuladores de gobierno analizados no presentan sistemas democráticos. Con una ausencia total de participación directa de la población en la toma de decisiones, las comunidades políticas virtuales se construyen sobre el consentimiento de la autoridad de sus líderes, sean estos escogidos o naturales, y sujetos a mayor o menor confrontación. Ese consentimiento se ve atenuado por una dimensión de contrapoder relativamente desarrollada, pero que en muchas ocasiones se enmarca como un problema técnico y dota de poca agencia (o directamente infantiliza) a los ciudadanos. Incluso en los juegos que buscan una representación más acertada (como Democracy), la actuación de los ciudadanos se construye sobre las bases de la teoría de la elección racional, lo que excluye cualquier tipo de aspecto deliberativo. Es complicado asociar los modelos de los simuladores de gobierno a experiencias democráticas reales. Si bien la importancia del consentimiento frente a la participación o la lógica del contrapoder nos acercaría más a planteamientos liberales que a, por ejemplo, el modelo de la Atenas clásica (Manin, 1998), estamos muy lejos de los primeros, dada la ausencia casi total de instituciones o la ten-

2 Las consecuencias de las decisiones de los obreros aparecen cómo eventos con efectos positivos o negativos para el objetivo del juego, según el caso. Sin embargo, el jugador pronto tiene una opción para firmar una ley que limita esa capacidad de actuación de los trabajadores y permite sólo decisiones con efectos positivos, lo que limita la importancia general de esa dinámica en el juego. 
dencia a presentar comunidades homogéneas. Aunque las propuestas de los simuladores beben de distintas fuentes, sus sistemas políticos son únicos y responden a las particularidades del videojuego y del género.

Este escenario es problemático en términos de valores transmitidos, dado que existen mecanismos ideológicos de justificación de la autocracia, pero existe espacio para mecánicas alternativas que refuercen valores prodemocráticos.

Este trabajo puede extenderse de distintas formas. Por una parte, requiere un análisis en mayor profundidad, que incorpore una dimensión narrativa y que también tenga en cuenta las percepciones de los jugadores. Otra línea de investigación podría encontrarse en el nivel del diseño, para intentar comprender cómo y por qué se han tomado las decisiones que han llevado a este tipo de representaciones (y que probablemente no sean consciente- mente ideológicas, sino que se expliquen por cosmovisiones subconscientes, principios del género y aproximaciones en términos de jugabilidad, mecánicas y desafíos). Pero la más interesante, sabiendo que existen representaciones problemáticas de la democracia en los videojuegos, probablemente sea proponer diseños alternativos, que nos lleven a pensar en los simuladores de gobierno de otra forma. Mecánicas relacionadas con ceder poder, dialogar, convencer o ser convencido, y escuchar y comprender podrían complementar la racionalización de los recursos de acuerdo con un objetivo y oponerse a la comprensión de los ciudadanos como recursos y oponentes, así como a la fantasía de poder que típicamente exhiben los videojuegos. Si los juegos pueden funcionar como escuelas de ciudadanía, tal vez puedan contribuir a replantear lo que significa la democracia, también para repensar nuestras propias sociedades. 


\section{REFERENCIAS}

Barthel, M. (2013). President for a day. Information, Communication \& Society, 16(1), 28-42, https://doi.org/10.1080/1369118X.2011.627176

Bogost, I. (2007). Persuasive Games: The Expressive Power of Videogames. MIT Press. https://doi.org/10.7551/mitpress/5334.001.0001

Centro de Investigaciones sociológicas (2021). Barómetro de febrero de 2021, estudio 3309.

Dishon, G., \& Kafai, Y. (2019): Connected civic gaming: rethinking the role of video games in civic education. Interactive Learning Environments, https://doi.org/10.1080/10494820.2019.1704791

Dolkemeyer, L. (2020). Autocracy for the People. Modes of response-able Action and the Management of Demise in Frostpunk. Gamevironments, 13. https://media.suub.uni-bremen.de/handle/elib/4605

Douglas, C. (2002). "You Have Unleashed a Horde of Barbarians!": Fighting Indians, Playing Games, Forming Disciplines. Postmodern culture, 13(1). https://doi.org/10.1353/pmc.2002.0029

Downs, A. (1957). An economic theory of democracy. Harper.

Evans-Thirlwell, E. (14 de agosto de 2021). SimCity wasn't built for the climate crisis. These games are. Wired. https://www.wired.co.uk/article/climatecrisis-city-building-games

Exmeyer, P., \& Boden, D. (2020). The 8-Bit Bureaucrat: Can Video Games Teach Us About Administrative Ethics? Public Integrity, 22(5), 1-16. https://doi.org/10.1080/10999922.2020.1731056

Ford, D. (2016). "explore, expand, exploit, exterminate": Affective Writing of Postcolonial History and Education in Civilization V. Game Studies, 16(2).

Habermas, J. (1998). Facticidad y validez. Trotta.

Imhoff, D., Godoy, J., Cena, M., \& Ferreira, P. (2021). Videojuegos y socialización política: evaluación del potencial de los videojuegos para promover aprendizajes sociopolíticos. Cuadernos del Centro de Estudios en Diseño y Comunicación, 130, 151-168.

Laclau, E. (2016). La razón populista. Fondo de Cultura Económica

Lang, A. (2007). But is it for real? The British Columbia Citizens' Assembly as a model of state-sponsored citizen empowerment. Politics \& Society, 35(1), 35-70.

Mair, P. (2015). Gobernando el vacío: la banalización de la democracia occidental. Alianza Editorial.

Manin, B. (1998). Los principios del gobierno representativo. Alianza Editorial. 
McHale, B. (1987): Postmodernist Fiction. Routledge.

McNeil, E. (2016). Ludic Spolia in Sid Meier's Civilization: Beyond Earth. Journal of games criticism. 3(1A).

Navarro Remesal, V. (2016). Libertad dirigida: una gramática del análisis y diseño de videojuegos. Shangrila.

Ober, J. (2018). Demópolis. La democracia antes del liberalismo: teoría y práctica. Casus-Belli.

Oliva, M., Besalú, R., \& Ciaurriz, F. (2009): Más grande, más rápido, mejor: la representación de la Historia universal en Civilization IV. Comunicación, $7(1), 62-79$.

Pérez Latorre, O. (2010). Análisis de la significación del videojuego. Fundamentos teóricos del juego, el mundo narrativo y la enunciación interactiva como perspectivas de estudio del discurso. [tesis doctoral, Universitat Pompeu Fabra].

Pfister, E., Winnerling, T., \& Zimmermann, F. (Eds). Democracy Dies Playfully. (Anti)Democratic Ideas in and Around Video Games. Gamevironments (2020, número especial).

Planells, A. (2015). Mundos posibles, grupos de presión y opinión pública en el videojuego Tropico 4. Trípodos, 37, 167-181.

Platón (2013). Ciudadanos políticamente competentes. En R. Aguila, F. Vallespín, A. Rivero, E. García, \& J. A. Pérez (Eds.) La democracia en sus textos, 38-42. Alianza Editorial.

Pobłocki, K. (2002). Becoming-state. Bio-cultural Imperialism of Sid Meier's Civilization. European Journal of Anthropology, 39, 163-177.

Schmitt, C. (1998). El concepto de lo político: texto de 1932 con un prólogo y tres corolarios. Alianza.

Schrier, K. (2016). Designing role-playing video games for ethical thinking. Educational Technology Research and Development, 65, 831-868.

Schrier, K. (2015). EPIC: a framework for using video games in ethics education. Journal of Moral Education, 44(4), 393-424, https://doi.org/10.1080 /03057240.2015.1095168

Schumpeter, J. (1971). Capitalismo, Socialismo y democracia. Aguilar.

Shields, M. (2009). A Pentadic Analysis of Tropico: Dramatism and Digital Games. [Tesis para el Master of Arts In Interdisciplinary Studies, Oregon State University]. https://tinyurl.com/exvjjd6n

Simkins, D. (2008). Critical Ethical Reasoning and Role-Play. Games and Culture, 3(3), 333-355. 
Tusell Collado, A. (2015): La calidad de la democracia y sus factores determinantes. Un análisis comparado de 60 países. Política y Sociedad, 52(1),179-204.

Zagal, J. P. (2011). Ethical reasoning and reflection as supported by single-player videogames. En K. Schrier, \& D. Gibson (Eds.), Designing Games for Ethics: Models, Techniques and Frameworks (pp. 19-35). IGI Global.

Zamaróczy, N. (2017). Are We What We Play? Global Politics in Historical Strategy Computer Games. International Studies Perspectives, 18(2), 155-174, https://doi.org/10.1093/isp/ekv010

\section{LUDOGRAFÍA (PC):}

Democracy 3 (2013). Positech Games, Positech Games

Endless Legend (2014). Amplitude Studios, SEGA

Faraón (1999). Impressions Games, Sierra Entertainment

Frostpunk (2018). 11 bit studios, 11 bit studios

Imperivm Civitas III (2009). Haemimont Games, FX Interactive

Sid Meier's Civilization V (2010). Firaxis Games, 2K Games

Surviving Mars (2018). Haemimont Games, Paradox Interactive

Surviving The Aftermath (2020). Iceflake Studios, Paradox Interactive

Tropico IV (2011). Haemimont Games, Kalypso Media 